

\title{
Derivative Based Algorithms for Continuous Relaxation Spectrum Recovery
}

\author{
R. S. Anderssen ${ }^{\mathrm{a}}$, A.R. Davies ${ }^{\mathrm{b}, *}$, F. R. de $\operatorname{Hoog}^{\mathrm{a}}$, R. J. Loy ${ }^{\mathrm{c}}$ \\ ${ }^{a}$ CSIRO Computational Informatics, GPO Box 664, Canberra, ACT 2601, Australia. \\ Electronic mail: Bob.Anderssen@csiro.au,Frank.deHoog@csiro.au \\ ${ }^{b}$ School of Mathematics, Cardiff University, Senghennydd Road, Cardiff CF24 4AG, UK. \\ Electronic mail: DaviesR@cardiff.ac.uk \\ ${ }^{c}$ Mathematical Sciences Institute, The Australian National University, John Dedman \\ Building, Union Lane, Canberra, ACT 2601, Australia. \\ Electronic mail: Rick.Loy@anu.edu.au
}

\begin{abstract}
Historically, it has always been understood that the relaxation spectra of linear viscoelastic materials are continuous. Nevertheless, because of their ease of implementation computationally, delta function recovery methods have been and continue to be important, even though they do not generate continuous approximations. Derivative based recovery techniques were popular in the precomputer days because they engendered simple formulas for continuous relaxation spectra approximation and estimation. They also represent a practical basis for continuous relaxation spectra estimation from oscillatory shear data. Here, using local Fourier deconvolution, we give precise formulae which generalize certain classical derivative based approximations to the relaxation spectra of linear viscoelastic materials using oscillatory shear data. We also present new formulae in this class. Finally we present a stable iterative algorithm, of the type proposed by Gureyev, which circumvents the calculation of very high order derivatives. The importance of the proposed derivative based approximations are that they are local and therefore are appropriate for the experimental situation where the oscillatory shear data is only available for a finite range of frequencies. Results are presented for both exact and experimental data.
\end{abstract}

Keywords: continuous relaxation spectrum, local Fourier deconvolution, Schwarzl-Staverman sequences, Maclaurin sequences, Gureyev iteration.

\section{Introduction}

To characterize and compare viscoelastic materials, it is common practice to use their relaxation time spectra, $H$, since estimates of these can be recovered

*Corresponding author. Electronic mail: DaviesR@cardiff.ac.uk 
from oscillatory shear measurements $[1,2,3,4,5]$, as well as step-strain and stepstress experiments [2]. The link between the spectra and measurements is the relaxation modulus, $G$, which takes the form

$$
G(t)=G_{e}+\int_{0}^{\infty} \exp (-t / \tau) \frac{H(\tau)}{\tau} d \tau=G_{e}+\int_{0}^{\infty} \exp (-t s) \frac{H(1 / s)}{s} d s,
$$

where $G_{e}=\lim _{t \rightarrow \infty} G(t)$ is a material constant which is zero for a viscoelastic liquid and non-zero for a viscoelastic solid. Estimates of $H$ are often modelled as finite sums of Dirac delta functions, with the corresponding relaxation modulus $G$ being finite (general) Dirichlet series of decaying exponential functions with positive coefficients $[6,7]$. However, this approach overlooks the facts that:

(a) In most oscillatory shear experiments, the measured stress-strain response is of a highly interconnected and cross-linked network of polymer chains [8] and not that of a simple network with minimal interconnectedness and cross-linking that one associates with an $H$ consisting of a finite sum of Dirac delta functions. The corresponding form for $G$, as a sum of decaying exponentials, may not capture the true decay of $G$ at large times [9].

(b) Historically, it has always been understood that the relaxation spectra of linear viscoelastic materials are continuous. This assumption can be validated in various ways. Jaishankar and McKinley [9] conclude that, in many situations, the storage and loss moduli have power law structures and mention the use of continuous models/approximations for $H$. As explained in Section 3, from a measure theory perspective, it is equally appropriate to assume that, for real viscoelastic liquids and solids, $H$ is a continuous function of compact support as it is to assume that it is a sum of point masses.

(c) Though the fading memory [10] of the relaxation modulus $G$ can be modelled using simpler relationships, it is common rheological practice to choose a form for $G$ that is a completely monotone function with $G$ assumed to be bounded in the neighbourhood of the origin. It therefore follows from Bernstein's theorem [11,12] that, on $[0, \infty), s^{-1} H\left(s^{-1}\right)$ must be a positive finite Borel measure. Within this class of measures, the absolutely continuous ones imply that $s^{-1} H\left(s^{-1}\right)$ is an $L_{1}$ function which is consistent with assuming that $H$ is continuous with compact support on $(0, \infty)$. For continuous $H$, because the span of the point masses (Dirac delta functions) is properly weak*-dense in the positive finite Borel measures [13], the number of point masses required to approximate the variable structure in $H$ can often be quite large $[6,7,9,14]$. The problematic nature of modelling $G$ with a large sum of decaying exponentials is discussed in Jaishankar and McKinley [9]

(d) Since not all completely monotone functions can be written as a finite (general) Dirichlet series with positive coefficients, such estimates for $H$ may not, in some practical situations, be representative of the actual characteristics of the viscoelastic material being studied. 
A quite detailed discussion about the practical limitations associated with approximating $H$ by a sum of Dirac delta functions as well about the historical use of continuous approximations for $H$ can be found in Stadler and Bailly [1]. It includes a discussion about the various merits and shortcomings of the different strategies that have been utilized for locating the Dirac delta functions.

Such considerations lead naturally to the need to have algorithms for constructing continuous approximations to $H$. This has been recognized by Stadler and Bailly [1] and Davies and Goulding [3]. In the Stadler and Bailly paper, $H$ is approximated by a piecewise cubic Hermite spline, a quadratically smooth approximation. They propose and analyse an algorithm that allows for the spline knots to be freely adjustable, in order to minimize the number of knots required to yield a functionally accurate recovery of the structure in the $H$ under investigation. Their motivation and justification for using spline approximations is that it avoids the explicit use of specific basis functions, such as the two-mode log-normal function proposed by Honerkamp and Weese [15]. In the Davies and Goulding paper (equation (3.7) in Section 3), $H$ is approximated by a sum of translated scaling functions (sometimes called kernel functions) $\phi_{\sigma, k}(t), k=1,2, \cdots, K$, located at appropriately chosen points $t_{k}, k=1,2, \cdots, K$,

$$
h_{\sigma}(t)=\sum_{k=1}^{K} a_{k} \phi_{\sigma, k}(t) \approx H(\exp (-t)), \quad \phi_{\sigma, k}(t)=\phi\left(\frac{t-t_{k}}{\sigma}\right),
$$

where $\phi$ denotes the chosen scaling function. Its potential advantage over the traditional discrete spectrum approaches is that, for a judicious choice for the scaling function, the number $K$ of terms required to yield a good approximation to $H$ will tend to be quite small. This is especially so if the determination of the locations of the $t_{k}, k=1,2, \cdots, K$, and the scaling function are orchestrated in an efficient manner.

The advantage in assuming that $H$ is continuous is that the possibility arises to use analytic methods and in particular, local methods, to construct approximation to $H$. An alternative to basis function approximation is the use of direct differentiation of the observational data. Using analytic methods, Schwarzl and Staverman [16], in 1952, were the first to introduce simple approximations based on derivatives, for the recovery of $H$ from measurements of $G$. The formulation of these earlier derivative formulas can be explained in the following way. For the chosen viscoelastic liquid, the starting point is a step-strain measurement of the relaxation modulus $G$. Since the corresponding $H$ is defined by equation (1.1), $\tau H(\tau)$ can be determined as the inverse Laplace transform of $G$. There are various ways in which this inversion can be performed [17,18].

As explained in Schwarzl and Staverman [16] the earlier methods proposed by Alfrey, Ferry and colleagues, either implicitly or explicitly, exploited the following inversion formula

$$
\tau H(\tau)=\lim _{n \rightarrow \infty}\left\{(-1)^{n} n^{n+1}(n !)^{-1} \tau^{(n+1)} G^{(n)}(n \tau)\right\}, \quad G^{(n)}(z)=\frac{d^{n} G(z)}{d z^{n}},
$$


The different formulae that they proposed corresponded to taking small values of $n$ in (1.3). As explained in Davies and Martin [17] (on page 6), the origin of this formula can be traced back to Gelfand and Shilov who were motivated by the idea of finding a transformation of $\exp (-t s)$ that produced an approximation to a Dirac delta function which could be applied directly to a Laplace transform equation. As mentioned in Davies and Martin, this formula was first published by Widder in 1934, but in a context independent of numerical Laplace transform inversion.

It is such endeavours that laid the foundation for the early popularity of derivative based recovery, especially since they generated continuous approximations for $H$. They represent motivation for the algorithms to be proposed below in that they correspond to the formulation and application of similar derivative based procedures to oscillatory shear measurements for the recovery of continuous approximations to $H$.

The paper has been organized in the following manner. Relevant aspects of continuous versus discrete relaxation spectrum recovery are discussed in Section 2. The Fourier deconvolution framework is defined in Section 3. The approximations and algorithms are defined, analysed and validated in Sections 4-7. Conclusions are drawn in Section 8.

\section{Continuous and discrete relaxation spectrum recovery}

Before turning to the formulation of derivative recovery algorithms that can be applied to oscillatory shear data to recover continuous approximations for the relaxation spectrum $H$, the significance of the relaxation spectrum $H$ being a positive finite Borel measure will be discussed, together with the implications of this for the construction of continuous approximations for $H$.

The relaxation modulus $G$ is assumed to be completely monotone $\mathcal{C} M[19]$. It therefore follows from Bernstein's theorem [11] that the most general form that the relaxation spectrum $H$ can have is as a positive finite Borel measure on $[0, \infty)$ such that $s^{-1} H\left(s^{-1}\right)$ is integrable. On the basis of the Lebesgue decomposition theorem [13], a general measure, $\mu$, on $[0, \infty)$ can be written uniquely as the sum

$$
\mu=\mu_{a c}+\mu_{s}, \quad \mu_{s}=\mu_{d}+\mu_{s c},
$$

where $\mu_{a c}$ denotes an absolutely continuous measure, while $\mu_{s}$ denotes a singular measure made up of a discrete measure $\mu_{d}$ and a singular continuous measure $\mu_{s c}$. From a measure theoretic perspective, it is necessary to draw a clear distinction between $\mu_{a c}$ and $\mu_{s}$, as $\mu_{s}$ is zero outside a set of Lebesgue measure zero, and $\mu_{a c}$ is given by the (Lebesgue) integral of some $L^{1}$ function.

The measure $\mu_{d}$, a discrete positive measure on $[0, \infty)$ has the form

$$
\mu_{d}=\sum_{i=1}^{\infty} \alpha_{i} \delta_{\tau_{i}}, \quad \tau_{i} \in[0, \infty), \quad \alpha_{i} \geq 0, \quad \sum_{i=1}^{\infty} \alpha_{i}<\infty
$$


where $\delta_{\tau}$ denotes the unit point mass located at $\tau \in[0, \infty)$. For theoretical reasons, in measure theory, it is often necessary to draw a distinction between discrete measures as above and the separated discrete measures that satisfy the additional condition that, for each $i$, distance $\left(\tau_{i} ;\left\{\tau_{j}: j \neq i\right\}\right)>0$. From a rheological perspective, it is the separated discrete that are used, not the nonseparated discrete, when generating discrete approximations to $H$.

As already mentioned, the span of the point masses is weak*-dense in the finite Borel measures. This is also true for continuous functions of compact support. The consequences of this have already been discussed in items (b) and (c) in the Introduction. In relation to the point masses, it follows, depending on the topology, that the span of the decaying exponentials, $S_{\text {DecayExp }}$, may not necessarily be dense in the space of $\mathcal{C} M$ functions. It is known that the denseness of $S_{\text {DecayExp }}$ holds for the pointwise and uniform topologies. For the $L_{p}$ topologies, it is only known that

$$
S_{\text {DecayExp }} \subset\left(\left(L_{p} \cup\{\text { constant functions }\}\right) \cap \mathcal{C M}\right) \text { for any } \mathrm{p}>0 .
$$

Consequently, on both theoretical and practical grounds, such results support the view that continuous $H$ should be approximated using the $\mu_{a c}$ measures.

\section{The oscillatory shear deconvolution framework}

As explained and exploited in Davies and Goulding [3], the recovery of $H(\tau)$ from measurements of the storage and loss moduli involves the solution of one or both of the following Fredholm integral equations of the first kind:

$$
G^{\prime}(\omega)=G_{e}+\int_{0}^{\infty} \frac{\omega^{2} \tau^{2}}{1+\omega^{2} \tau^{2}} H(\tau) \frac{d \tau}{\tau}, \quad G^{\prime \prime}(\omega)=\int_{0}^{\infty} \frac{\omega \tau}{1+\omega^{2} \tau^{2}} H(\tau) \frac{d \tau}{\tau}
$$

These equations can be reformulated in terms of a Fourier deconvolution problem: for given data $g$ and known kernel $k$, solve the Fourier convolution integral equation

$$
g=k * h, \quad k * h=\int_{-\infty}^{\infty} k(x-y) h(y) d y=\int_{-\infty}^{\infty} h(x-y) k(y) d y,
$$

for $h$. For viscoelastic liquids, on applying the following substitutions

$$
h(x)=H(\exp (-x)), \quad g_{1}(x)=2 G^{\prime}(\exp (x)), \quad g_{2}(x)=2 G^{\prime \prime}(\exp (x)),
$$

to the oscillatory shear equations (3.1), the corresponding counterparts of equation (3.2) become

$$
\begin{aligned}
& g_{1}(x)=[1+\tanh (x)] * h(x), \\
& g_{2}(x)=\operatorname{sech}(x) * h(x) .
\end{aligned}
$$


Upon differentiating (3.4), the solutions of these convolution equations may be expressed as

$$
\begin{aligned}
h(t) & \left.=\lim _{\epsilon \rightarrow 0} \frac{1}{2 \pi^{2}} \int_{-\infty}^{\infty} \int_{-\infty}^{\infty} \frac{d g_{1}(x)}{d x} p^{-1} \sinh \left(\frac{\pi}{2} p\right) \exp \left(-\frac{1}{2} \epsilon^{2} p^{2}\right) \exp (i p(t-x))\right) d p d x, \\
h(t) & \left.=\lim _{\epsilon \rightarrow 0} \frac{1}{2 \pi^{2}} \int_{-\infty}^{\infty} \int_{-\infty}^{\infty} g_{2}(x) \cosh \left(\frac{\pi}{2} p\right) \exp \left(-\frac{1}{2} \epsilon^{2} p^{2}\right) \exp (i p(t-x))\right) d p d x,
\end{aligned}
$$

where

(i) the mollifier $\exp \left(-\frac{1}{2} \epsilon^{2} p^{2}\right)$ has been introduced to ensure the convergence of the inner integrals, and

(ii) it is assumed that the functions $g_{1}$ and $g_{2}$ have sufficient regularity to ensure all limit operations are interchangeable.

In physical variables these equations represent the inversion formulae

$$
\begin{aligned}
& H\left(\tau^{-1}\right)=\lim _{\epsilon \rightarrow 0} \frac{1}{\pi^{2}} \int_{-\infty}^{\infty} \int_{-\infty}^{\infty} \frac{d G^{\prime}(\omega)}{d \ln \omega} p^{-1} \sinh \left(\frac{\pi}{2} p\right) \exp \left(-\frac{1}{2} \epsilon^{2} p^{2}\right) \exp (-i p \ln \omega \tau) d p d \ln \omega \\
& H\left(\tau^{-1}\right)=\lim _{\epsilon \rightarrow 0} \frac{1}{\pi^{2}} \int_{-\infty}^{\infty} \int_{-\infty}^{\infty} G^{\prime \prime}(\omega) \cosh \left(\frac{\pi}{2} p\right) \exp \left(-\frac{1}{2} \epsilon^{2} p^{2}\right) \exp (-i p \ln \omega \tau) d p d \ln \omega
\end{aligned}
$$

On replacing the sinh and cosh terms by their Maclaurin expansions, performing the double integration term by term and taking the limit $\epsilon \rightarrow 0$, the following analytic expressions for $H$ in terms of $G^{\prime}$ or in terms of $G^{\prime \prime}$ can be derived:

$$
\begin{aligned}
H\left(\omega^{-1}\right) & =\frac{2}{\pi} \sum_{r=0}^{\infty}(-1)^{r}\left(\frac{\pi}{2}\right)^{2 r+1} \frac{1}{(2 r+1) !}\left[\frac{d^{2 r+1} G^{\prime}(\omega)}{d(\ln \omega)^{2 r+1}}\right], \\
& =\frac{2}{\pi} \sum_{r=0}^{\infty}(-1)^{r}\left(\frac{\pi}{2}\right)^{2 r} \frac{1}{(2 r) !}\left[\frac{d^{2 r} G^{\prime \prime}(\omega)}{d(\ln \omega)^{2 r}}\right] .
\end{aligned}
$$

Although the above series appear to be new, the unpublished $\mathrm{PhD}$ thesis of Al-Aidarous [20] contains similar results, derived using an operational method based on repeated differentiation. The series could also be derived using the more general (Fourier) deconvolution procedure given in Gureyev et al. [21].

Using the substitutions in (3.3), with $x=\ln \omega$, the equations (3.10) and (3.11) become

$$
\begin{aligned}
h(x) & =\frac{1}{\pi} \sum_{r=0}^{\infty}(-1)^{r}\left(\frac{\pi}{2}\right)^{2 r+1} \frac{1}{(2 r+1) !}\left[\frac{d^{2 r+1} g_{1}(x)}{d x^{2 r+1}}\right], \\
& =\frac{1}{\pi} \sum_{r=0}^{\infty}(-1)^{r}\left(\frac{\pi}{2}\right)^{2 r} \frac{1}{(2 r) !}\left[\frac{d^{2 r} g_{2}(x)}{d x^{2 r}}\right] .
\end{aligned}
$$




\section{The Schwarzl-Staverman sequence and the Maclaurin sequence.}

The first approximations to the continuous relaxation spectrum using derivatives of the storage and loss moduli appeared in a classic paper of Schwarzl and Staverman [22], a sequel to their 1952 paper. Their method of derivation was based on intensity functions which lead to delta-sequences, i.e. sequences of functions of decreasing width and increasing height which approach the Dirac delta function in the limit. These ideas were pursued further by Tschoegl [23] and developed within a more general framework by Friedrich [24].

Starting from the inversion formulae (3.8) and (3.9) it is possible to derive a sequence of higher order approximations involving odd derivatives of the storage modulus and even derivatives of the loss modulus which extend the method of Schwarzl and Staverman to arbitrary order. Similar results are to be found in Friedrich [24] in a more general algebraic setting. Our approach here leads to a much simpler formulation of the results. In (3.8) and (3.9) we take the infinite-product representations

$$
\sinh \left(\frac{\pi}{2} p\right)=\frac{\pi}{2} p \prod_{r=1}^{\infty}\left(1+\frac{p^{2}}{(2 r)^{2}}\right), \quad \cosh \left(\frac{\pi}{2} p\right)=\prod_{r=1}^{\infty}\left(1+\frac{p^{2}}{(2 r-1)^{2}}\right)
$$

and consider their approximation by the polynomials obtained by expanding the first $\mathrm{n}$ factors under the product sign in each case:

$$
\begin{array}{r}
\sinh \left(\frac{\pi}{2} p\right) \approx \frac{\pi}{2} p \prod_{r=1}^{n}\left(1+\frac{p^{2}}{(2 r)^{2}}\right)=\frac{\pi}{2} \sum_{r=0}^{n} a_{2 r+1}^{(n)} p^{2 r+1} \\
\cosh \left(\frac{\pi}{2} p\right) \approx \prod_{r=1}^{n}\left(1+\frac{p^{2}}{(2 r-1)^{2}}\right)=\sum_{r=0}^{n} a_{2 r}^{(n)} p^{2 r}
\end{array}
$$

The coefficients $a_{0}^{(n)}$ and $a_{1}^{(n)}$ are equal to 1 for all $n$, while the coefficients $a_{r}^{(n)}, r=2, \ldots, n$ depend on $n$, and are clearly positive and rational. Substituting (4.2) and (4.3) into (3.8) and (3.9), respectively, and evaluating the integrals term by term leads to the approximations

$$
\begin{aligned}
H_{2 n+1}^{S S}\left(\omega^{-1}\right) & =\sum_{r=0}^{n}(-1)^{r} a_{2 r+1}^{(n)} \frac{d^{2 r+1} G^{\prime}(\omega)}{d(\ln \omega)^{2 r+1}} \\
H_{2 n}^{S S}\left(\omega^{-1}\right) & =\frac{2}{\pi} \sum_{r=0}^{n}(-1)^{r} a_{2 r}^{(n)} \frac{d^{2 r} G^{\prime \prime}(\omega)}{d(\ln \omega)^{2 r}} .
\end{aligned}
$$


The first few approximations in the sequence $\left\{H_{n}^{S S}(\tau)\right\}_{n=0}^{\infty}$ are:

$$
\begin{aligned}
& H_{0}^{S S}(\tau)=\frac{2}{\pi} G^{\prime \prime}\left(\tau^{-1}\right) \\
& H_{1}^{S S}(\tau)=D G^{\prime}\left(\tau^{-1}\right) \\
& H_{2}^{S S}(\tau)=\frac{2}{\pi}\left[G^{\prime \prime}\left(\tau^{-1}\right)-D^{2} G^{\prime \prime}\left(\tau^{-1}\right)\right] \\
& H_{3}^{S S}(\tau)=D G^{\prime}\left(\tau^{-1}\right)-\frac{1}{4} D^{3} G^{\prime}\left(\tau^{-1}\right)
\end{aligned}
$$

where $D$ denotes the differential operator $d / d l n \omega$. The approximation (4.6) is usually attributed to Fuoss and Kirkwood [8], while (4.7) - (4.9) were first derived by Schwarzl and Staverman [22]. They did not derive formulae of order higher than three in this sequence, but in recognition of their pioneering work we shall refer to the sequence of approximations $\left\{H_{n}^{S S}(\tau)\right\}_{n=0}^{\infty}$ as the SchwarzlStaverman (SS) sequence.

From the independent perspective of derivative spectroscopy [25], the derivative terms can be viewed as recovering information about the changing structure in $H$ that is hidden in the oscillatory shear data. Given exact data, it may be shown that

(A) the accuracy of each approximation increases with $n$;

(B) every approximation in the sequence satisfies the glassy modulus constraint

$$
\int_{-\infty}^{\infty} H(\tau) d \ln \tau=\frac{2}{\pi} \int_{-\infty}^{\infty} G^{\prime \prime}(\omega) d \ln \omega=G^{\prime}(\infty),
$$

which holds for the exact spectrum $H$;

(C) the sequence is a sequence of positive functions [20].

Another sequence, of a different form to the SS-sequence, can be obtained by truncating the infinite series (3.10) and (3.11). We refer to this new sequence, $\left\{H_{n}^{M}(\tau)\right\}_{n=0}^{\infty}$, whose entries are given by the following formulae, as the Maclaurin sequence or M-sequence:

$$
\begin{aligned}
H_{2 n+1}^{M}\left(\omega^{-1}\right) & =\frac{2}{\pi} \sum_{r=0}^{n}(-1)^{r}\left(\frac{\pi}{2}\right)^{2 r+1} \frac{1}{(2 r+1) !}\left[\frac{d^{2 r+1} G^{\prime}(\omega)}{d(\ln \omega)^{2 r+1}}\right] \\
H_{2 n}^{M}\left(\omega^{-1}\right) & =\frac{2}{\pi} \sum_{r=0}^{n}(-1)^{r}\left(\frac{\pi}{2}\right)^{2 r} \frac{1}{(2 r) !}\left[\frac{d^{2 r} G^{\prime \prime}(\omega)}{d(\ln \omega)^{2 r}}\right] .
\end{aligned}
$$

The approximations in the SS-sequence and the M-sequence have the same zeroth and first order coefficients, but the higher-order coefficients are different. The coefficients under the summation sign in the M-sequence are no longer rational, but are rational multiples of powers of $\pi$. The properties (A) and (B) which hold for the SS-sequence also hold for the M-sequence, but low-order 
approximations in the M-sequence may display small negative lobes. The size of these lobes diminish quite quickly with increasing $n$. We shall return to this point later.

We shall demonstrate in the following section that the M-sequence converges much faster than the SS-sequence. It can take derivatives of order twelve in the SS-sequence to give the same accuracy as given by derivatives of fourth order in the M-sequence.

\section{Comparison of SS- and M-sequences}

In this section we compare the convergence of the SS- and M-sequences. Due to the property (A) shared by both sequences it will be sufficient to compare the even-order subsequences (4.5) and (4.12). Schwarzl and Staverman [22] used a single delta-function test spectrum to facilitate comparison of different approximations. However, since the delta-function is a point mass, and the approximations in the SS-sequence are continuous, they were unable to make precise quantitative comparisons. We choose instead a continuous test spectrum which is the unimodal function

$$
H(\tau)=\frac{2 \tau^{2}}{1+\tau^{4}}
$$

The corresponding storage and loss moduli are given by

$$
G^{\prime}(\omega)=\frac{2 \omega^{2}}{1+\omega^{4}}\left(\frac{\pi}{4} \omega^{2}-\ln \omega\right), \quad G^{\prime \prime}(\omega)=\frac{\pi}{\sqrt{2}} \frac{\omega}{1+\omega^{4}}\left(1-\sqrt{2} \omega+\omega^{2}\right) .
$$

The spectrum is of unit height with a maximum at $\tau=1$. The corresponding functions $h(x), g_{1}(x)$ and $g_{2}(x)$, defined by the substitutions (3.3), are given by

$$
\begin{aligned}
h(x) & =\operatorname{sech}(2 \mathrm{x}), \\
g_{1}(x) & =\frac{\pi}{2}[1+\tanh (2 x)]-2 x \operatorname{sech}(2 \mathrm{x}), \\
g_{2}(x) & =\pi \operatorname{sech}(2 \mathrm{x})[\sqrt{2} \cosh (x)-1] .
\end{aligned}
$$

The function $h(x)$ is symmetric with its maximum at $x=0$. For the purposes of comparison, all differentiations are carried out exactly using MAPLE 17 . We adopt the notation $h_{n}^{S S}(x)$ to denote $H_{n}^{S S}\left(e^{-x}\right)$ and $h_{n}^{M}(x)$ to denote $H_{n}^{M}\left(e^{-x}\right)$.

In Figure 1, the zeroth, second-order and fourth-order approximations to $h(x)$ in the SS-sequence are shown, together with the exact spectrum for comparison. The function $h(x)$ is shown in black, and $h_{0}^{S S}(x), h_{2}^{S S}(x)$ and $h_{4}^{S S}(x)$ are shown in green, blue and red, respectively. The convergence of the sequence to the true spectrum is slow, with the fourth-order approximation achieving a peak-height of only $75 \%$. 


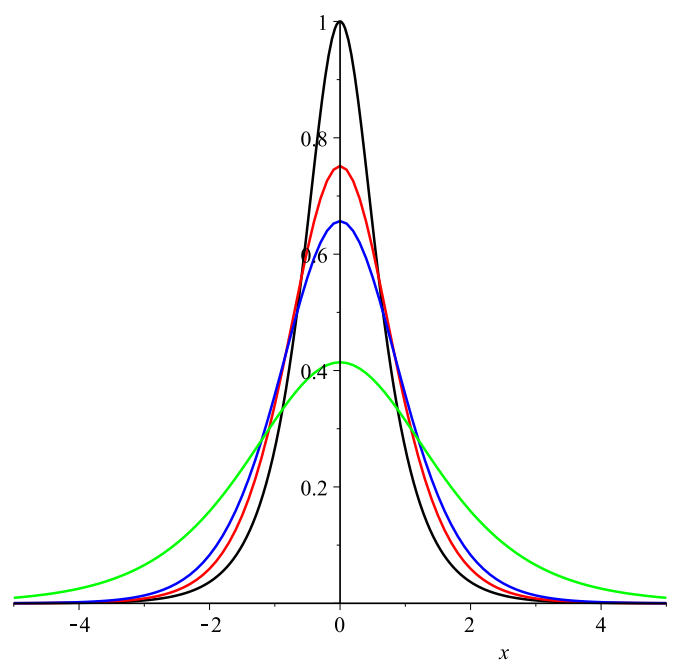

Figure 1: $h(x)\left(\_\right), h_{0}^{S S}(x)\left(\_\right), h_{2}^{S S}(x)\left(\_\right), h_{4}^{S S}(x)\left(\_\right)$.

In Figure 2, the zeroth, second-order and fourth-order approximations to $h(x)$ in the M-sequence are shown, using the same colour scheme. The second-order approximation $h_{2}^{M}(x)$ has negative lobes of magnitude $0.5 \%$ of its peak height. For the test spectrum under consideration, all M-approximations higher than second-order are positive. The fourth-order approximation $h_{4}^{M}(x)$ achieves a peak-height of $87 \%$. In the SS-sequence it takes a twelfth-order approximation $h_{12}^{S S}(x)$ to reach a peak-height of $86 \%$.

Figure 3 shows the sixth-order approximations in both sequences, with $h_{6}^{M}(x)$ shown in red. The approximation $h_{6}^{M}(x)$ is clearly much superior, achieving a peak-height of $94 \%$.

\section{Gureyev iteration}

Stable methods for the numerical differentation of observational data can be found in the papers by Anderssen, de Hoog and Hegland [26,27,28]. However, in practice, working with experimental data, it would be prohibitively challenging to employ derivative-based formulae of order higher than 2 or 3 . It is clear that the M-sequence and the SS-sequence, regardless of their mathematical elegance, are not practicable when working with experimental data, due to the high orders of differentiation required to provide sufficient accuracy in the recovery of the spectrum. We must therefore find a way of capturing the structure of the spectrum hidden in the higher derivatives without direct computation of these derivatives. 


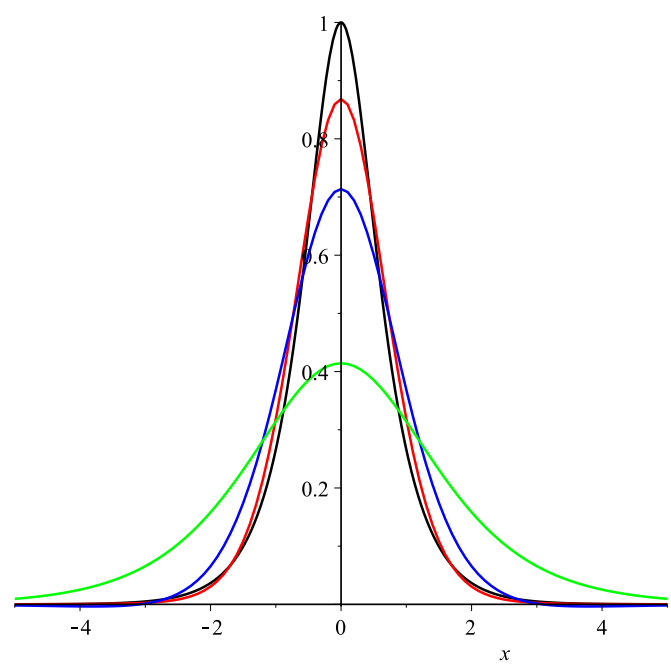

Figure 2: $h(x)\left(\_\right), h_{0}^{M}(x)\left(\_\right), h_{2}^{M}(x)\left(\_\right), h_{4}^{M}(x)\left(\_\right)$.

To achieve this we adapt an iterative technique originally proposed by Gureyev [21]. When recovering the spectrum from the loss modulus, this involves repeated application of the second-order differential operator in the Msequence. This process effectively replaces high-order derivatives of the data by a sequence of convolutions involving second order derivatives of the sech-kernel.

We take the second order differential operator $L=\frac{1}{\pi}-\frac{\pi}{8} D^{2}$, defined by the approximation

$$
h_{2}^{M}(x)=L g_{2}(x),
$$

and apply the Gureyev iteration

$$
\begin{aligned}
& h_{2,0}^{M}(x)=h_{2}^{M}(x) ; \\
& h_{2, k}^{M}(x)=h_{2, k-1}^{M}(x)+L\left[g_{2}(x)-\operatorname{sech}(\mathrm{x}) * h_{2, k-1}^{M}(x)\right], \quad k=1,2, \ldots .
\end{aligned}
$$

The great advantage of this approach is that we may use the identity

$$
L[\operatorname{sech}(\mathrm{x}) * h(x)]=[L \operatorname{sech}(\mathrm{x})] * h(x)
$$

to replace high-order derivatives of the data by a sequence of convolutions with the function $L \operatorname{sech}(\mathrm{x})$. This function may be evaluated exactly, and acts as a smoothing operator under convolution. In consequence, the iterations are stable in the presence of experimental noise. The convergence of the Gureyev iteration is much less rapid than the convergence of the M-sequence. Fortunately, this is no great setback since each iteration in (6.3) may be performed very quickly, with 100 iterations being achievable in about 1 second on a modest computer. 


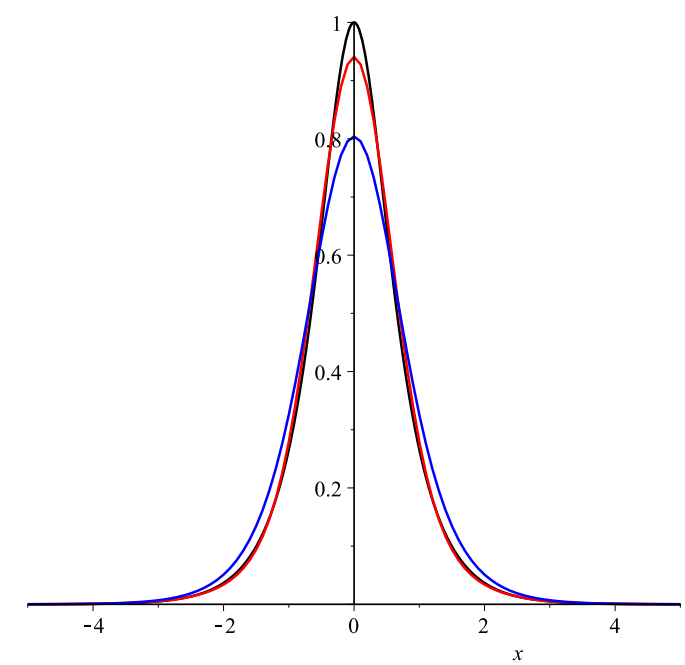

Figure 3: $h(x)\left(\_\right), h_{6}^{S S}(x)\left(\_\right), h_{6}^{M}(x)\left(\_\right)$.

Applying this iteration to the M-approximation $h_{2}^{M}(x)$, the negative lobes are removed after only a few iterations, and a recovery of the positive test spectrum to a peak-height of $98 \%$ is achieved in 48 iterations. The approximation $h_{2,48}^{M}(x)$ is shown in red in Figure 4, and the initial estimate $h_{2}^{M}(x)$ is shown in blue.

To recover the spectrum from the storage modulus we must start from the third-order approximation in the M-sequence. We take the differential operator $K=\frac{1}{2}-\frac{\pi^{2}}{48} D^{2}$, defined by the approximation

$$
h_{3}^{M}(x)=K \frac{d g_{1}(x)}{d x},
$$

and apply the Gureyev iteration

$$
\begin{aligned}
& h_{3,0}^{M}(x)=h_{3}^{M}(x) ; \\
& h_{3, k}^{M}(x)=h_{3, k-1}^{M}(x)+K\left[\frac{d g_{1}(x)}{d x}-\operatorname{sech}^{2}(\mathrm{x}) * h_{3, k-1}^{M}(x)\right], \quad k=1,2, \ldots .
\end{aligned}
$$

We again use the identity

$$
K\left[\operatorname{sech}^{2}(\mathrm{x}) * h(x)\right]=\left[K \operatorname{sech}^{2}(\mathrm{x})\right] * h(x)
$$

to circumvent repeated differentiation of the data.

Starting from the third-order approximation (6.6), the iteration defined by (6.7) recovers the spectrum to a peak height of $98 \%$ after only 17 iterations. It 


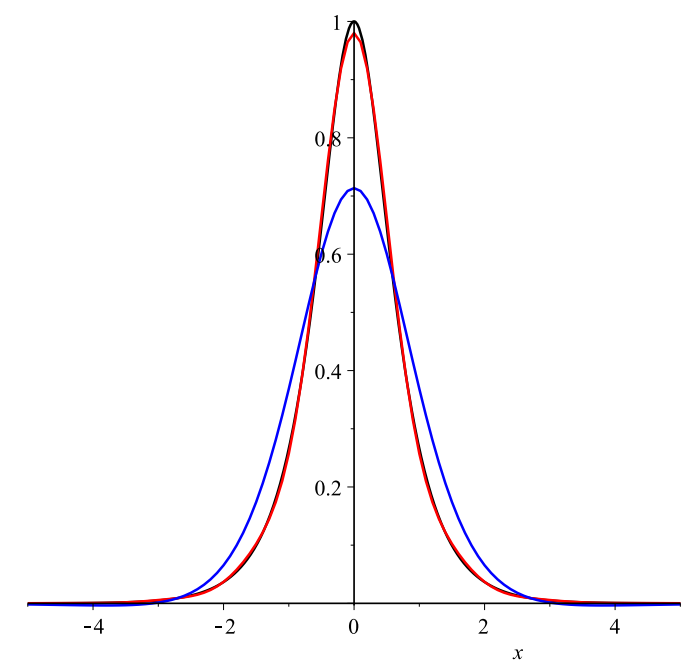

Figure 4: Spectrum recovery from the loss modulus using Gureyev iteration. $h(x)\left(\_\right), h_{2}^{M}(x)$ $\left(\right.$ ( ), $h_{2,48}^{M}(x)($ ( ).

obtains the same accuracy as the iteration defined by (6.3) in fewer iterations (17 rather than 48) since the starting point is a better approximation than the second-order approximation given by (6.2). The results are shown in Figure 5. The two recovered spectra shown in red in Figures 4 and 5 differ by less than $0.1 \%$ of their peak height.

\section{Working with experimental data}

The problem of recovering the relaxation spectrum from dynamic data is well-known to be ill-posed. Ill-posed inverse problems abound in the natural sciences, and fall into one of three categories: mildly ill-posed, moderately illposed, and severely ill-posed. Davies and Goulding [3] show that the deconvolution problems described by equations (3.4) and (3.5) are in fact exponentially ill-posed. Consequently they fall into the severe category.

Working with experimental data, which are naturally discrete, noisy, and confined to a limited frequency range, poses far greater challenges than those encountered in earlier sections where we have worked with exact data over the full frequency range. In this section we use experimental data published by Honerkamp and Weese [29] for a polybutadiene blend, which we refer to as PBD1. This choice of data enables us to compare results generated by our derivative-based algorithms with published results obtained by other methods.

For ease of reference, we call the scheme of iterations given by equations (6.2)-(6.3) the loss algorithm, and the scheme of iterations given by equations (6.6)-(6.7) the storage algorithm. The loss algorithm generates a spectrum from 


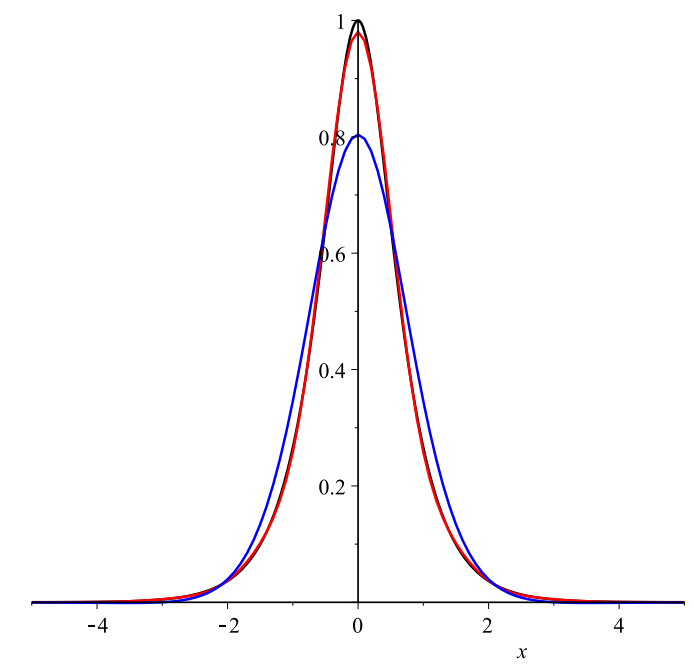

Figure 5: Spectrum recovery from the storage modulus using Gureyev iteration. $h(x)(-)$, $h_{3}^{M}(x)\left(\right.$ ( ) , $h_{3,17}^{M}(x)($ ( ) .

$G^{\prime \prime}$-data alone, whereas the storage algorithm determines a spectrum from $G^{\prime}$ data alone. Since the experimental data in themselves cannot be expected to satisfy the Kramers-Kronig relations

$$
\begin{gathered}
G^{\prime}(\omega)=G_{\infty}^{\prime}-\frac{2}{\pi} P V \int_{0}^{\infty} \frac{\omega_{1} G^{\prime \prime}\left(\omega_{1}\right)}{\omega_{1}^{2}-\omega^{2}} d \omega_{1}, \\
G^{\prime \prime}(\omega)=\frac{2}{\pi} P V \int_{0}^{\infty} \frac{\omega G^{\prime}\left(\omega_{1}\right)}{\omega_{1}^{2}-\omega^{2}} d \omega_{1},
\end{gathered}
$$

the spectra generated by each algorithm will be different. We shall show that it is possible to combine the two estimates to best effect.

The experimental PBD1-data is shown in Figure 6, together with two underlying analytic curves which are generated for the purpose of providing estimates of derivatives up to order 2 in the case of $G^{\prime \prime}$ and order 3 in the case of $G^{\prime}$. There are only 17 frequency values, spanning less than 3 decades. These values are in the range $2.413 s^{-1}<\omega<1114 s^{-1}$, or $0.91<\ln \omega<7.02$. The central frequency value is $\omega_{0}=52.18 s^{-1}$.

The analytic $G^{\prime \prime}$-curve is generated as follows. The full log-frequency range $-\infty<\ln \omega<\infty$ is shrunk onto the finite interval $-1<y<1$ using the map

$$
y=\tanh \left[\lambda \ln \left(\frac{\omega}{\omega_{0}}\right)\right],
$$

where $\lambda$ is an adjustable parameter which controls the smoothness of the higher derivatives of both $G^{\prime \prime}$ and $G^{\prime}$. A suitable value of $\lambda$ will depend on the data 


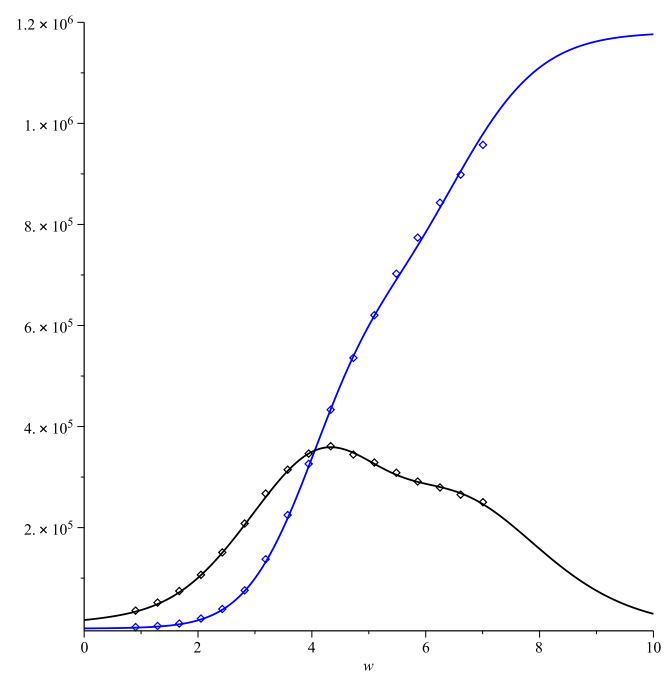

Figure 6: The PBD1 data and their analytic representations: $G^{\prime \prime}(\ldots) . G^{\prime}(\ldots)$. Horizontal axis: $w=\ln \omega$.

set under consideration. For the PBD1 data the value $\lambda=0.3$ is chosen. In the $y$-variable, $G^{\prime \prime}$ is given the representation

$$
G^{\prime \prime}(\omega)=\sum_{r=0}^{m} a_{r} \alpha_{r}(y),
$$

where

$$
\alpha_{r}(y)=(1+y)^{r}\left(1-y^{2}\right)^{2},
$$

and the $m+1$ coefficients $a_{r}$ are determined by fitting (7.4) to the $G^{\prime \prime}$-data by weighted least-squares regression. The basis functions (7.5) are chosen to give realistic asymptotics for $G^{\prime \prime}$ in the limit of low and high frequencies. In terms of $\ln \omega$ the $G^{\prime \prime}$-curve is a sum of powers of hyperbolic functions. The $G^{\prime \prime}$-curve in Figure 6 corresponds to a value of $m=6$, and the goodness-of-fit is marked by a root-mean-square (RMS) error of $1 \%$.

The $G^{\prime}$-curve is generated in a similar way. $G^{\prime}$ is given the representation

$$
G^{\prime}(\omega)=\sum_{r=0}^{m} b_{r} \beta_{r}(y),
$$

where

$$
\beta_{r}(y)=\int_{s=-1}^{y} \alpha_{r}(s) d s .
$$

Again, the $m+1$ coefficients $b_{r}$ are determined from the $G^{\prime}$-data by weighted least-squares regression, but with one important constraint. An estimate of 


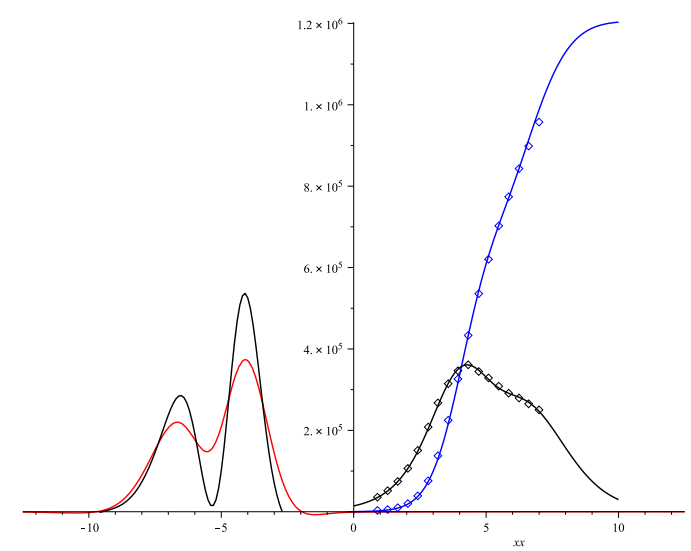

Figure 7: Results for PBD1 from the loss algorithm. Left: Initial estimate for the spectrum (_). Converged spectrum after 20 iterations (_). Horizontal axis: $\ln \tau$. Right: Experimental $G^{\prime \prime}(\ldots) . G^{\prime \prime}$ obtained from the spectrum (_). Experimental $G^{\prime}(\ldots) . G^{\prime}$ obtained from the spectrum (_). Horizontal axis: $\ln \omega$.

the limiting modulus, $G_{\infty}^{\prime}$, must be included in the regression. Without this additional constraint, the $G^{\prime}$-curve will not be Kramers-Kronig compatible with the $G^{\prime \prime}$-curve. Consequently the $G^{\prime \prime}$ data generated from the spectrum obtained by the storage algorithm will not coincide with the experimental $G^{\prime \prime}$ values over their full measured range.

To circumvent this problem the $G^{\prime \prime}$-curve is calculated first and the limiting modulus estimated from the formula

$$
G_{\infty}^{\prime}=\frac{2}{\pi} \int_{-\infty}^{\infty} G^{\prime \prime}(\omega) d \ln \omega .
$$

The $G^{\prime}$-curve shown in Figure 6 corresponds to a value of $m=6$ in equation (7.6), and carries an RMS error of $1.2 \%$. The limiting modulus estimate is $G_{\infty}^{\prime}=1.2 \times 10^{6}$.

We first implement the loss algorithm, using the $G^{\prime \prime}$-curve in Figure 6 to obtain the second derivative of $G^{\prime \prime}$ required. The initial estimate for the spectrum is obtained from equations (6.1)-(6.2) using the substitution $g_{2}(x)=G^{\prime \prime}\left(e^{x}\right)$, and is depicted by the red curve on the left of Figure 7. Only 20 iterations are required for numerical convergence to the spectrum shown in black on the left of Figure 7. The first 10 iterations deliver a spectrum with a small negative end-lobe. For the final 10 iterations the support of the spectrum is restricted to that interval for which the spectrum in iteration 10 is positive. This is sufficient to deliver the positive spectrum shown.

On the right of Figure 7 is shown the $G^{\prime}$ - and $G^{\prime \prime}$-curves reproduced from the converged spectrum using equations (3.4) and (3.5), respectively. These curves satisfy the Kramers-Kronig relations to within machine precision since 


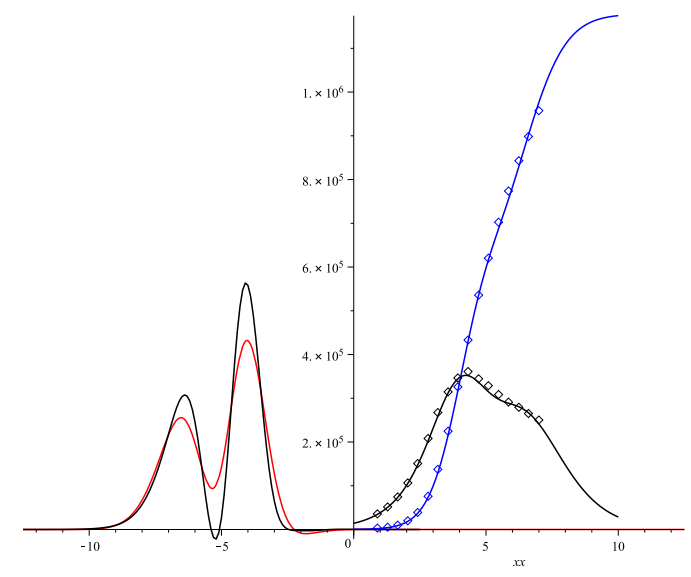

Figure 8: Results for PBD1 from the storage algorithm. Left: Initial estimate for the spectrum (—). Converged spectrum after 10 iterations (-). Horizontal axis: $\ln \tau$. Right: Experimental $G^{\prime \prime}(\ldots) . G^{\prime \prime}$ obtained from the spectrum (_). Experimental $G^{\prime}(\ldots) . G^{\prime}$ obtained from the spectrum (_). Horizontal axis: $\ln \omega$.

they are generated from the same spectrum. They are plotted together with the experimental data. The RMS error of fit to the $G^{\prime \prime}$-data is $1.7 \%$, whereas the RMS error of fit to the $G^{\prime}$-data is $3.0 \%$. The higher error in the latter case is due to the fact that only $G^{\prime \prime}$-data is used by the loss algorithm to generate the spectrum.

We next implement the storage algorithm, using the $G^{\prime}$-curve in Figure 6 to obtain the first and third derivatives of $G^{\prime}$ required. The initial estimate for the spectrum is obtained from equations (6.5)-(6.6) using the substitution $g_{1}(x)=$ $G^{\prime}\left(e^{x}\right)$, and is depicted by the red curve on the left of Figure 8. Numerical convergence to the spectrum shown in black on the left of Figure 8 is obtained after 10 iterations. This spectrum has a small negative region in its central dip, and is therefore not acceptable. Nevertheless, a much improved result may be found by combining the two spectra in Figures 7 and 8, thereby making use of both $G^{\prime}$ - and $G^{\prime \prime}$-data.

A straight average (50\% weighting in each case) of the two spectra produce the positive spectrum shown on the left of Figure 9. The $G^{\prime}$ - and $G^{\prime \prime}$-curves on the right of Figure 9 are generated from the combined spectrum, and again satisfy the Kramers-Kronig relations to within machine precision. The RMS error of fit to the $G^{\prime \prime}$-data is $1.7 \%$, whereas the RMS error of fit to the $G^{\prime}$-data is reduced to $1.8 \%$. The reduced error in the latter case is due to the fact that both $G^{\prime}$ - and $G^{\prime \prime}$-data are used to generate the combined spectrum.

The combined spectrum obtained in Figure 9 is superior to that found by Tikhonov regularization (see Figure 17 of Ref [3]). The combined spectrum is also very similar to that found for PBD1 by the method of wavelet regularization 


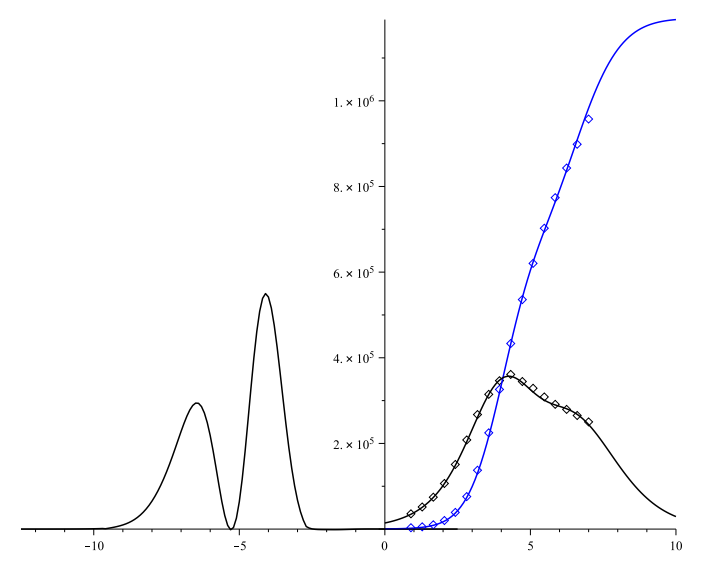

Figure 9: Results for PBD1 from the combined spectrum. Left: The combined spectrum (—). Horizontal axis: $\ln \tau$. Right: Experimental $G^{\prime \prime}(\ldots) . G^{\prime \prime}$ obtained from the spectrum $(-)$. Experimental $G^{\prime}$ (...). $G^{\prime}$ obtained from the spectrum (_). Horizontal axis: $\ln \omega$.

in Figure 16 of Ref [3]. If the criterion of overall RMS error in reproducing the $G^{\prime}$ - and $G^{\prime \prime}$-data is used for the purpose of comparison, then the results in Figure 9 show an overall RMS error of $1.75 \%$ while those obtained by wavelet regularization show an overall RMS error of $1.8 \%$. The improvement is hardly significant, but what is significant is the simplicity of the algorithms used to generate the results in the current paper compared with the method of wavelet regularization. It is clear from Ref [3] that the choice of scale, vital to the success of wavelet regularization, is a non-trivial exercise.

\section{Conclusions}

The topic of higher derivative approximations for recovery of the continuous relaxation spectrum goes back several decades to pre-computer days. In this paper we have revisited some of the early ideas and developed them further in a modern context. In particular, the complete Schwarzl-Staverman sequence for recovering the spectrum from derivatives of the dynamic data has been expressed in a simple format and compared with a new sequence derived from Maclaurin series. Although the Schwarzl-Staverman sequence has inbuilt positivity of the spectrum guaranteed, at least when working with high quality data, we have demonstrated that this sequence converges very slowly compared with the Maclaurin sequence. This is easily explained by comparing the rate of convergence of the infinite products (4.1) with their corresponding Maclaurin expansions. The fact that positivity is not an inbuilt property of the Maclaurin sequence is not a setback. Small negative lobes, which can appear in very 
low-order Maclaurin approximations, disappear once higher order terms are included, provided that the true spectrum is smooth.

It has been demonstrated that higher derivatives of order 6 and above are needed to achieve accurate recovery of the spectrum. This is not practicable when working with experimental data, and consequently algorithms which circumvent the computation of derivatives higher than order 2 or 3 are needed. We have advocated the use of Gureyev iteration, which replaces high-order differentiation with a sequence of well-defined convolutions, and we have shown that this algorithm is capable of approximating the spectrum with high accuracy in the case of exact data.

Spectrum approximations are computable from both of the moduli $G^{\prime}$ and $G^{\prime \prime}$ separately. When working with experimental data, this leads to different estimates of the spectrum. In Section 7 it has been demonstrated that these two estimates can be combined to advantage. This is consistent with the experience reported by others, that, when determining relaxation spectra from experimental data, using both $G^{\prime}$ - and $G^{\prime \prime}$-data leads to better results than working with $G^{\prime}$ - and $G^{\prime \prime}$-data separately.

Acknowledgements. Part of this work was carried out when ARD visited CSIRO Canberra. ARD is grateful for the facilities and financial assistance provided during his visit.

\section{References}

1. Stadler FJ, Bailly C (2009). A new method for the calculation of continuous relaxation spectra from dynamic-mechanical data. Rheol Acta 48:3349.

2. Ferry JD (1980). Viscoelastic properties of polymers. Wiley, New York.

3. Davies AR, Goulding NJ (2012). Wavelet regularization and the continuous relaxation spectrum. J. Non-Newtonian Fluid Mech. 189:19-30.

4. Tanner RI, Walters K (1998). Rheology: An Historical Perspective. Elsevier, Amsterdam.

5. Walters K (1975). Rheometry. Chapman and Hall, London.

6. Baumgaertel M, Winter HH (1989). Determination of Discrete Relaxation and Retardation Time Spectra from Dynamic Mechanical Data. Rheol Acta 28:511-519.

7. Baumgaertel M, Winter HH (1992). Interrelation between continuous and discrete relaxation time spectra. J Non-Newton Fluid Mech 44:15-36.

8. Fuoss R, Kirkwood J (1941). Electrical Properties of Solids. VIII. Dipole Moments in Polyvinyl Chloride-Diphenyl Systems. J Amer Chem Soc. 63:385-394.

9. Jaishankar A, McKinley GH (2013). Power-law rheology in the bulk and at the interface: quasi-properties and fractional constitutive equations. Proc. Roy. Soc. A. Volume 469 Issue 2149 Article Number 20120284 Published Jan 82013 . 
10. Saut JC, Joseph DD (1983). Fading memory. Arch. Rational. Mech. Anal. 81:53-95.

11. Bernstein SN (1928). Sur les fonctions absolument monotones. Acta Mathematica 52: 1-66.

12. Loy RJ, Anderssen RS (2014). On the construction of Dirichlet series approximations for completely monotone functions. Math. Comp. 83:835846.

13. Megginson RE (1998). An introduction to Banach space theory. Springer, New York.

14. Tschoegl NW (1989). The phenomenological theory of linear viscoelastic behavior: an introduction. Springer-Verlag, Berlin.

15. Honerkamp J, Weese, J (1993). A nonlinear regularization method for the calculation of relaxation spectra. Rheol Acta 32:65-73.

16. Schwarzl F, Staverman AJ (1952). Higher approximations of relaxation spectra. Physica 18:791-798.

17. Davies B, Martin B (1979). Numerical inversion of the Laplace transform - Survey and comparison of methods. J. Comp. Phys. 33:1-32.

18. de Hoog FR, Knight JH, Stokes AN (1982). An improved method for numerical inversion of Laplace transforms. SIAM J. Sci. Stat. Computing 3:357-366.

19. Anderssen RS, Loy RJ (2002). Rheological implications of completely monotone fading memory. J Rheol 46:1459-1472.

20. Al-Aidarous ES (2005). The numerical inversion of complex Hilbert transform. PhD thesis, University of Wales Aberystwyth.

21. Gureyev TE, Nesterets YI, Stevenson AW, Wilkins SW (2003). A method for local deconvolution. Applied Optics 42:6488-6494.

22. Schwarzl F, Staverman AJ (1953). Higher approximation methods for the relaxation spectrum from static and dynamic measurements of visco-elastic materials. Appl. Sci. Res A 4:127-141.

23. Tschoegl NW (1973). A general method for the determination of approximations to the spectral distributions from the dynamic response functions. Rheol Acta 12:82-83.

24. Friedrich C (1991). A delta-function method for the $n$-th approximation of relaxation or retardation time spectrum from dynamic data. Rheol Acta 30:7-13.

25. Anderssen R, Hegland (2010). Derivative spectroscopy - An enhanced role for numerical differentiation. J. Integ. Eqn. Appl. 22:355-367.

26. Anderssen RS, de Hoog FR (1984). Finite-difference methods for the numerical differentiation of non-exact data. Computing 33:259-267.

27. Anderssen RS, de Hoog FR, Hegland M (1998). A stable finite difference ansatz for higher order differentiation of non-exact data. Bull. Austral. Math. Soc. 58:223-232.

28. Anderssen RS, Hegland M (1999). For numerical differentiation, dimensionality can be a blessing! Math. Comp. 68:1121-1141.

29. Honerkamp J, Weese J (1989). Determination of the relaxation spectrum by a regularization method. Macromolecules 22:4372-4377. 